\title{
Occurrence and Sources of Aliphatic Hydrocarbons in Soils within the Vicinity of Hot Mix Asphalt Plants in Obigbo and Igwuruta Areas of Rivers State, Nigeria
}

\section{*11 IFENNA ILECHUKWU; ${ }^{2}$ LEO C. OSUJI; ${ }^{2}$ MARK O. ONYEMA; ${ }^{3}$ GLORIA I. NDUKWE}

\author{
${ }^{1}$ Department of Industrial Chemistry, Madonna University, P.M.B 48, Elele, Rivers State, Nigeria \\ ${ }^{2}$ Department of Pure and Industrial Chemistry, University of Port Harcourt, P.M.B 5323, Choba, Port Harcourt, Nigeria. \\ ${ }^{3}$ Department of Chemistry, Rivers State University of Science and Technology, Nkpolu-Oroworukwo \\ P.M.B. 5080 Port Harcourt, Rivers State, Nigeria \\ ifennai@yahoo.com
}

\begin{abstract}
This study investigated the occurrence and sources of aliphatic hydrocarbons (AHCs) in soils within the vicinity of hot mix asphalt (HMA) plants. Soil samples were collected from two HMA plants and analysed with gas chromatography - flame ionisation detector (GC-FID). Sample collection was from both surface $(0-15 \mathrm{~cm})$ and subsurface $(15-30 \mathrm{~cm})$ soils at an increasing distance of $10 \mathrm{~m}$ from the HMA plants while the control samples at both depths were collected at $1 \mathrm{~km}$ away from the plants. The AHCs concentration $\left(n \mathrm{C}_{9}-n \mathrm{C}_{38}\right)$ in soils from both HMA Plants ranged from $8.16-433.04 \mathrm{mg} / \mathrm{kg}$ and $5.91-177.83 \mathrm{mg} / \mathrm{kg}$ for surface and subsurface soils respectively while the concentration in the control samples ranged from $5.97-23.95 \mathrm{mg} / \mathrm{kg}$ and $2.52-13.31$ for surface and subsurface soil respectively. The sources of the AHCs were determined using isoprenoid ratios, carbon preference index (CPI) and maximum carbon chain $\left(\mathrm{C}_{\max }\right)$. The isoprenoid ratios and CPI values of the samples closer $(<20 \mathrm{~m})$ to the HMA plants were $<1$ and $<2$ respectively indicating anthropogenic inputs. Samples $>20 \mathrm{~m}$ from the HMA plants showed mixture of both anthropogenic and biogenic sources. CJASEM
\end{abstract}

http://dx.doi.org/10.4314/jasem.v20i4.24

Keywords: Asphalt Plants; Soil; Aliphatic hydrocarbons; Pristane; Phytane.

Hot mix asphalt (HMA) plant is an assemblage of mechanical equipment where aggregates or inert mineral materials such as sand, gravel, crushed stones, Slag, rock dust or powder are blended, heated, dried and mixed with bitumen in measured quantities (Mamlouk and Zaniewski, 2011). It can be constructed as a permanent plant, a skid-mounted (easily relocated) plant, or a portable plant (EPA, 2000). HMA plants can be a source of a number of basic environmental pollutants. These pollutants may issue from dryers, burner blowers, exhaust fans and dust collection systems (Rilwanu and Agbanure, 2010). Other activities like asphalt loading, use and disposal of petroleum products as well as routine vehicle and plant maintenance also contribute to environmental pollution sources within HMA plant vicinities (Ilechukwu, 2013).

Petroleum hydrocarbons ranging from light products like diesel to heavy residues such as bitumen are used in asphalt production process. These petroleum products have variable hydrocarbon composition and distribution that differ from each other (Wang et al., 2004). For example, bitumen, a product of nondestructive distillation of crude oil consisting of aliphatic and cyclic alkanes, aromatic hydrocarbons, heterocyclic compounds as well as metals like nickel, vanadium and iron serves as the cement that binds the aggregate materials (NIOSH, 2001). These petroleum hydrocarbons may be released into the environment from spills, indiscriminate waste disposal, atmospheric depositions and vehicular emissions. Source determination of these hydrocarbons in environmental media makes it possible to identify, differentiate, determine risk to health and environment, direct remedial efforts and assign blame where necessary (Yang et al., 2011; Ziegler et al., 2008; Zemo et al., 2007; Kassim, 2005).

Aliphatic hydrocarbons (AHCs) are ubiquitous environmental contaminants. They consist of odd and even carbon numbers ranging up to 64 carbons with no alkyl branch or substitutes. They have been employed in source determination of hydrocarbon pollution especially in distinguishing biogenic hydrocarbons from petrogenic hydrocarbons. Wang et al., (1995) reported that the biogenic hydrocarbons have characteristic chemical composition where the aliphatic hydrocarbons show a distribution pattern of odd-carbon-numbered aliphatics being much abundant than even carbon-numbered aliphatics in the range of $n \mathrm{C}_{21}-n \mathrm{C}_{33}$. The "even" carbon numbers are from anthropogenic sources while the "odd" carbons are usually derived from the biogenic sources especially from terrestrial vascular plants. (Sakari et al. 2008; Azevedo et al., 2007). The objective of this study therefore, was to determine the occurrence and sources of aliphatic hydrocarbons in soils within the vicinity of HMA plants.

\section{MATERIALS AND METHODS}

The two HMA plants used for this study were located in Obigbo (HMA Plant A) and Igwuruta (HMA Plant B) areas of Rivers State, Nigeria. HMA Plant A is located between Latitude ( $4^{\circ} 51^{\prime} 0$ " and $\left.4^{\circ} 51^{\prime} 55^{\prime \prime}\right) \mathrm{N}$ and longitudes ( $7^{\circ} .04$ '55" and $7^{\circ} .05$ '26") E while HMA Plant B is located between Latitude ( $4^{\circ} 55^{\prime} 04^{\prime \prime}$ and $\left.4^{\circ} 56^{\prime} 30^{\prime \prime}\right) \mathrm{N}$ and longitudes $\left(7^{\circ} .02\right.$ ' $30^{\prime \prime}$ and 
$7^{\circ} .03$ ' 0") E. Both plants have been in operation for 12 years and are in a sparsely residential area

surrounded by farmlands.

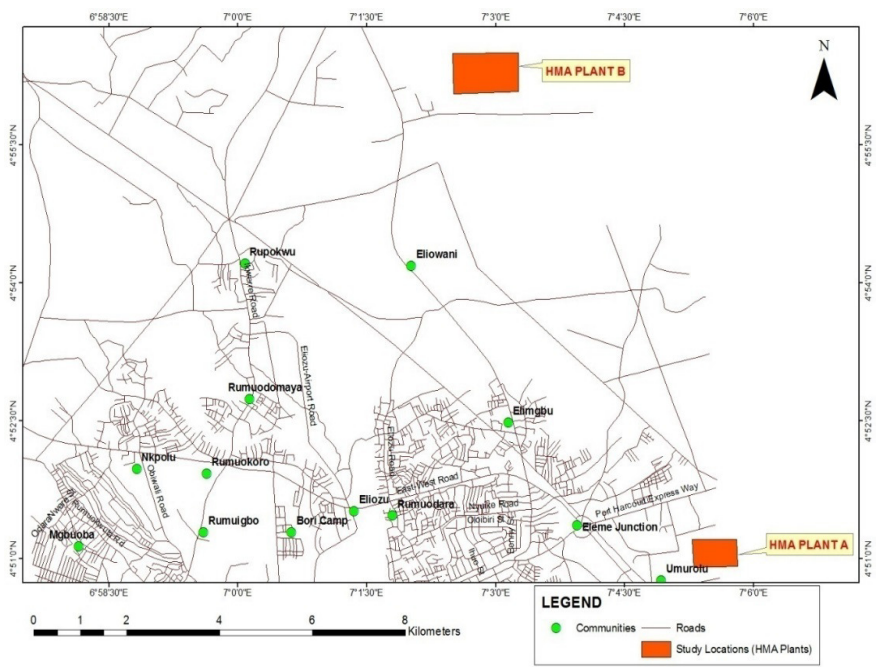

Fig. 1: Map of the Study Area indicating the HMA Plants

Soil samples were collected in March (dry season) and August (rainy season). Samples were collected at both surface $(0-15 \mathrm{~cm})$ and subsurface $(15-30 \mathrm{~cm})$ depths. They were randomly collected across the plant vicinities and blended to form a homogenous mix. This was done at an increasing distance of $10 \mathrm{~m}$ from both plants terminating at $50 \mathrm{~m}$ and $40 \mathrm{~m}$ for Plant A and Plant B respectively. The control samples were collected at $1 \mathrm{~km}$ away from both plants.

Ten grams (10 g) of each soil sample was extracted in a soxhlet apparatus for $4 \mathrm{~h}$ with $200 \mathrm{ml}$ of dichloromethane. The extracts were concentrated to 1 $\mathrm{ml}$ using a rotary evaporator. Each of the concentrated extract was fractionated using an activated silica gel column (100-200 mesh, $15 \times 1$ $\mathrm{cm}$ ) topped with $0.5 \mathrm{~g}$ anhydrous sodium sulphate for water removal. The column was eluted with $20 \mathrm{ml}$ of hexane to obtain the aliphatic fractions. Each of these fractions was concentrated to $1 \mathrm{ml}$ using a rotary evaporator.

One microlitre $(1 \mu \mathrm{l})$ of each sample extract was analysed with AGILENT 6890 gas chromatography equipped with flame ionization detector (FID) under the following operational conditions: capillary column of length $25.0 \mathrm{~m}$, diameter $320 \mu \mathrm{m}$, stationary phase; phenyl methylsiloxane, film thickness 0.17 $\mu \mathrm{m}$. The column temperature was programmed from $40^{\circ} \mathrm{C}$ to $320^{\circ} \mathrm{C}$ at a rate of $6^{\circ} \mathrm{C} / \mathrm{min}$ and held at $350^{\circ} \mathrm{C}$ for 15 minutes. Helium was used as the carrier gas.
The injector was maintained at $325^{\circ} \mathrm{C}$ and $1 \mu 1$ of each of the sample extract was injected in splitless mode.

Quantification was determined by comparing the peak area of the internal standard perdeuterated ntetracosane to those of the analytes. The total aliphatic hydrocarbons for each extract were quantified by GC/FID via total detector response.

\section{RESULTS AND DISCUSSION}

The concentration of aliphatic hydrocarbons in the soil samples from HMA Plant A is shown in Tables 1 and 2 for dry and rainy seasons respectively while that of HMA Plant B is shown in Tables 3 and 4 for dry and rainy seasons respectively.

The AHCs concentration in soils from HMA Plant A during the dry season ranged from 9.81 - 433.04 $\mathrm{mg} / \mathrm{kg}$ and $6.76-177.83 \mathrm{mg} / \mathrm{kg}$ for surface and subsurface soils respectively while the concentration in the control samples was $5.97 \mathrm{mg} / \mathrm{kg}$ and 2.52 $\mathrm{mg} / \mathrm{kg}$ for surface and subsurface soils respectively. In the rainy season, the concentration of the AHCs ranged from $8.16-82.85 \mathrm{mg} / \mathrm{kg}$ and $7.43-27.36$ $\mathrm{mg} / \mathrm{kg}$ for surface and subsurface soils respectively while the concentration in the control samples was $7.85 \mathrm{mg} / \mathrm{kg}$ and $5.86 \mathrm{mg} / \mathrm{kg}$ for surface and subsurface soils respectively. 
Table 1: Aliphatic hydrocarbons concentration in HMA Plant A (Dry Season)

\begin{tabular}{|c|c|c|c|c|c|c|c|c|c|c|c|c|}
\hline \multirow{2}{*}{$\begin{array}{c}\text { AHCs } \\
(\mathrm{mg} / \mathrm{kg})\end{array}$} & \multicolumn{2}{|c|}{$10 \mathrm{~m}$} & \multicolumn{2}{|c|}{$20 \mathrm{~m}$} & \multicolumn{2}{|c|}{$30 \mathrm{~m}$} & \multicolumn{2}{|c|}{$40 m$} & \multicolumn{2}{|c|}{$50 \mathrm{~m}$} & \multicolumn{2}{|c|}{$1 \mathrm{KM}$} \\
\hline & $\mathbf{S}$ & SS & $\mathbf{S}$ & SS & $\mathbf{S}$ & SS & $\mathbf{S}$ & SS & $\mathbf{S}$ & SS & $\mathrm{CS}$ & CSS \\
\hline C9 & 0.00 & 0.00 & 0.00 & 0.00 & 0.00 & 0.00 & 0.00 & 0.00 & 0.00 & 0.00 & 0.00 & 0.00 \\
\hline $\mathrm{C} 10$ & 0.00 & 0.00 & 0.00 & 0.00 & 0.00 & 0.00 & 0.00 & 0.00 & 0.00 & 0.00 & 0.00 & 0.00 \\
\hline $\mathrm{C} 11$ & 0.00 & 5.73 & 0.00 & 0.00 & 0.00 & 0.00 & 0.00 & 0.00 & 0.00 & 0.00 & 0.00 & 0.00 \\
\hline $\mathrm{C} 12$ & 11.36 & 5.60 & 0.00 & 0.00 & 0.00 & 1.13 & 1.32 & 0.00 & 0.00 & 0.00 & 0.00 & 0.00 \\
\hline $\mathrm{C} 13$ & 5.64 & 7.08 & 0.00 & 0.00 & 0.00 & 0.34 & 1.09 & 0.00 & 0.00 & 0.00 & 0.00 & 0.00 \\
\hline $\mathrm{C} 14$ & 11.45 & 8.37 & 1.44 & 0.00 & 1.12 & 0.74 & 1.54 & 0.00 & 0.00 & 0.00 & 0.00 & 0.00 \\
\hline $\mathrm{C} 15$ & 17.76 & 7.92 & 0.91 & 0.00 & 0.00 & 1.35 & 1.79 & 0.00 & 0.00 & 0.00 & 0.00 & 0.00 \\
\hline C16 & 33.88 & 15.66 & 2.65 & 2.14 & 0.00 & 1.45 & 13.90 & 0.00 & 0.00 & 0.00 & 0.00 & 0.00 \\
\hline $\mathrm{C} 17$ & 22.56 & 15.35 & 3.53 & 1.80 & 3.29 & 0.00 & 0.00 & 0.00 & 0.00 & 0.00 & 0.00 & 0.00 \\
\hline Pristane & 13.25 & 8.82 & 1.41 & 1.40 & 0.00 & 0.00 & 0.00 & 0.00 & 0.00 & 0.00 & 0.00 & 0.00 \\
\hline C18 & 21.74 & 8.07 & 6.18 & 1.74 & 1.39 & 0.00 & 0.00 & 0.00 & 0.00 & 0.00 & 0.00 & 0.00 \\
\hline Phytane & 14.31 & 2.33 & 2.44 & 0.28 & 0.00 & 0.00 & 0.00 & 0.00 & 0.00 & 0.00 & 0.00 & 0.00 \\
\hline C19 & 10.83 & 9.36 & 7.58 & 2.26 & 1.72 & 0.00 & 0.00 & 0.00 & 0.00 & 0.00 & 0.00 & 0.00 \\
\hline $\mathrm{C} 20$ & 26.36 & 7.69 & 2.68 & 1.07 & 1.79 & 0.56 & 0.00 & 0.59 & 0.56 & 1.42 & 0.00 & 0.58 \\
\hline $\mathrm{C} 21$ & 18.86 & 9.47 & 1.72 & 0.47 & 1.40 & 0.55 & 0.43 & 0.58 & 1.56 & 0.00 & 0.00 & 0.56 \\
\hline $\mathrm{C} 22$ & 9.60 & 3.50 & 2.92 & 6.72 & 1.52 & 2.01 & 0.00 & 0.00 & 0.00 & 0.00 & 0.00 & 0.00 \\
\hline $\mathrm{C} 23$ & 9.37 & 7.89 & 3.91 & 0.67 & 1.65 & 1.70 & 0.00 & 0.00 & 1.46 & 0.00 & 0.00 & 0.00 \\
\hline $\mathrm{C} 24$ & 9.85 & 3.26 & 0.78 & 1.26 & 0.77 & 0.93 & 0.00 & 0.95 & 1.94 & 0.00 & 0.00 & 0.00 \\
\hline $\mathrm{C} 25$ & 9.09 & 2.58 & 1.00 & 0.85 & 0.88 & 0.00 & 0.00 & 0.00 & 0.00 & 0.00 & 0.00 & 0.00 \\
\hline $\mathrm{C} 26$ & 2.96 & 4.30 & 1.52 & 0.94 & 1.17 & 0.00 & 0.00 & 0.00 & 0.00 & 0.00 & 0.00 & 0.00 \\
\hline $\mathrm{C} 27$ & 14.82 & 5.11 & 1.66 & 1.08 & 1.54 & 1.35 & 0.00 & 1.41 & 4.29 & 1.41 & 0.00 & 1.38 \\
\hline $\mathrm{C} 28$ & 15.80 & 2.71 & 1.97 & 1.18 & 2.02 & 0.00 & 1.29 & 0.00 & 0.00 & 0.00 & 0.00 & 0.00 \\
\hline $\mathrm{C} 29$ & 12.84 & 3.97 & 1.91 & 1.50 & 1.71 & 0.00 & 1.37 & 1.02 & 0.00 & 0.00 & 1.39 & 0.00 \\
\hline C30 & 30.80 & 8.53 & 6.39 & 2.41 & 4.37 & 0.00 & 1.43 & 0.00 & 0.00 & 0.00 & 0.00 & 0.00 \\
\hline C31 & 27.26 & 5.02 & 2.10 & 1.55 & 1.96 & 0.00 & 0.00 & 0.00 & 0.00 & 0.00 & 2.22 & 0.00 \\
\hline C32 & 13.04 & 1.57 & 1.31 & 0.00 & 1.57 & 0.00 & 0.00 & 0.00 & 0.00 & 0.00 & 0.00 & 0.00 \\
\hline C33 & 29.72 & 2.54 & 1.38 & 0.00 & 1.40 & 0.00 & 0.00 & 0.00 & 0.00 & 0.00 & 0.00 & 0.00 \\
\hline C34 & 9.34 & 1.70 & 1.40 & 0.00 & 0.00 & 0.00 & 0.00 & 0.00 & 0.00 & 1.32 & 0.00 & 0.00 \\
\hline C35 & 5.26 & 3.55 & 1.97 & 2.13 & 3.19 & 3.49 & 1.95 & 2.27 & 0.00 & 2.61 & 2.36 & 0.00 \\
\hline C36 & 5.25 & 1.70 & 1.67 & 0.00 & 0.00 & 0.00 & 0.00 & 0.00 & 0.00 & 0.00 & 0.00 & 0.00 \\
\hline C37 & 8.49 & 3.54 & 1.93 & 0.00 & 0.00 & 0.00 & 0.00 & 0.00 & 0.00 & 0.00 & 0.00 & 0.00 \\
\hline C38 & 11.55 & 4.91 & 2.28 & 0.00 & 0.00 & 0.00 & 0.00 & 0.00 & 0.00 & 0.00 & 0.00 & 0.00 \\
\hline TOTAL & 433.04 & 177.83 & 66.64 & 31.45 & 34.46 & 15.60 & 26.11 & 6.82 & 9.81 & 6.76 & 5.97 & 2.52 \\
\hline Pri/Phy & 0.93 & 3.79 & 0.58 & 4.99 & nd & nd & nd & nd & nd & nd & nd & nd \\
\hline $\mathrm{CPI}_{25-35}$ & 1.22 & 0.86 & 0.64 & 1.10 & 0.76 & 1.44 & 0.50 & 2.57 & 0.69 & nd & 0.52 & nd \\
\hline $\mathrm{C}_{\max }$ & C16 & C16 & C19 & $\mathrm{C} 22$ & $\mathrm{C} 30$ & C35 & C16 & C35 & $\mathrm{C} 27$ & C35 & $\mathrm{C} 35$ & $\mathrm{C} 27$ \\
\hline C17/Pri & 1.70 & 1.74 & 2.50 & 1.29 & nd & nd & nd & nd & nd & nd & nd & nd \\
\hline C18/Phy & 1.52 & 3.46 & 2.53 & 6.21 & nd & nd & nd & nd & nd & nd & nd & nd \\
\hline
\end{tabular}

In HMA Plant B, the concentration of the AHCs in the dry season ranged from $99.02-389.84 \mathrm{mg} / \mathrm{kg}$ and $5.91-116.22 \mathrm{mg} / \mathrm{kg}$ for surface and subsurface soils respectively while the concentration in the control sample was $23.95 \mathrm{mg} / \mathrm{kg}$ and $9.14 \mathrm{mg} / \mathrm{kg}$ for surface and subsurface soils respectively. In the rainy season, the range was between $39.03-65.08 \mathrm{mg} / \mathrm{kg}$ and 8.69 - $33.34 \mathrm{mg} / \mathrm{kg}$ for surface and subsurface soils respectively while the concentration in the control samples was $14.76 \mathrm{mg} / \mathrm{kg}$ and $13.31 \mathrm{mg} / \mathrm{kg}$ for surface and subsurface soils respectively.

The AHCs concentration decreased with increase in distance from HMA Plant $\mathrm{A}$, an indication that the plant is the dominant source of AHCs in the vicinity. The AHCs concentration in all the samples was higher than that of the control samples except subsurface sample $20 \mathrm{~m}$ in HMA Plant B which was lower than the corresponding control subsurface sample in both seasons. Furthermore, the concentration of the AHCs in the surface soils was higher than that of the corresponding subsurface soils at all sampling location. This also indicates that the AHCs might be migrating downward the soil profile from the top soil. Unlike HMA Plant A, there was no steady decline in the concentration of AHCs in HMA Plant B as the distance from the Plant increases. This could be attributed to the fact that Plant B also serves as an equipment/heavy duty vehicle yard. The maintenance and repair of these equipment and vehicles also contributed to the hydrocarbon content of the soils within the HMA plant vicinity.

The general decrease in the AHCs concentration in the samples from both plants during the rainy season may be attributed to dissolutions and runoffs from rainfall as well as a significant decrease in asphalt production during the rainy season due to reduced construction activities. 
Table 2: Aliphatic hydrocarbons concentration in HMA Plant A (Rainy Season)

\begin{tabular}{|c|c|c|c|c|c|c|c|c|c|c|c|c|}
\hline \multirow{2}{*}{$\begin{array}{c}\text { AHCs } \\
(\mathrm{mg} / \mathrm{kg})\end{array}$} & \multicolumn{2}{|c|}{$10 \mathrm{~m}$} & \multicolumn{2}{|c|}{$20 \mathrm{~m}$} & \multicolumn{2}{|c|}{$30 \mathrm{~m}$} & \multicolumn{2}{|c|}{$40 m$} & \multicolumn{2}{|c|}{$50 \mathrm{~m}$} & \multicolumn{2}{|c|}{$1 \mathrm{KM}$} \\
\hline & $\mathbf{S}$ & SS & $\mathbf{S}$ & SS & $\mathbf{S}$ & SS & $\mathbf{S}$ & SS & $\mathbf{S}$ & SS & CS & CSS \\
\hline C9 & 0.00 & 0.00 & 0.00 & 0.00 & 0.00 & 0.00 & 0.00 & 0.00 & 0.00 & 0.00 & 0.00 & 0.00 \\
\hline $\mathrm{C} 10$ & 0.00 & 0.00 & 0.00 & 0.00 & 0.00 & 0.00 & 0.00 & 0.00 & 0.00 & 0.00 & 0.00 & 0.00 \\
\hline $\mathrm{C} 11$ & 0.00 & 0.00 & 0.00 & 0.00 & 0.00 & 0.00 & 0.00 & 0.00 & 0.00 & 0.00 & 0.00 & 0.00 \\
\hline $\mathrm{C} 12$ & 0.00 & 0.00 & 0.00 & 0.00 & 0.00 & 0.00 & 0.00 & 0.00 & 0.00 & 0.00 & 0.00 & 0.00 \\
\hline $\mathrm{C} 13$ & 0.00 & 0.00 & 0.00 & 0.00 & 0.00 & 0.00 & 0.00 & 0.00 & 0.00 & 0.00 & 0.00 & 0.00 \\
\hline $\mathrm{C} 14$ & 12.21 & 6.13 & 0.00 & 0.00 & 0.00 & 0.00 & 1.34 & 1.70 & 1.06 & 1.28 & 0.00 & 1.12 \\
\hline $\mathrm{C} 15$ & 0.00 & 0.00 & 1.43 & 0.00 & 0.00 & 0.00 & 0.00 & 0.00 & 0.00 & 0.00 & 0.00 & 0.00 \\
\hline $\mathrm{C} 16$ & 1.15 & 0.00 & 1.69 & 0.00 & 0.00 & 0.00 & 0.00 & 0.00 & 0.00 & 0.00 & 0.00 & 0.00 \\
\hline $\mathrm{C} 17$ & 3.61 & 0.00 & 1.39 & 1.60 & 1.68 & 0.00 & 0.00 & 0.00 & 0.00 & 0.00 & 1.86 & 0.00 \\
\hline Pristane & 13.68 & 11.4 & 3.48 & 1.40 & 1.29 & 1.06 & 1.48 & 1.80 & 1.68 & 1.23 & 1.02 & 1.77 \\
\hline $\mathrm{C} 18$ & 6.65 & 0.00 & 1.51 & 1.08 & 0.62 & 1.10 & 3.16 & 0.00 & 3.62 & 0.00 & 0.84 & 0.00 \\
\hline Phytane & 16.23 & 3.00 & 4.15 & 1.50 & 1.01 & 1.85 & 3.60 & 1.65 & 1.80 & 1.06 & 1.16 & 1.75 \\
\hline C19 & 9.51 & 1.21 & 0.00 & 0.00 & 0.00 & 1.69 & 0.00 & 0.00 & 0.00 & 1.13 & 1.14 & 0.00 \\
\hline $\mathrm{C} 20$ & 1.54 & 3.93 & 1.09 & 1.36 & 2.51 & 1.13 & 0.00 & 2.32 & 0.00 & 1.66 & 1.83 & 0.00 \\
\hline $\mathrm{C} 21$ & 0.00 & 0.00 & 0.00 & 1.71 & 0.99 & 1.32 & 1.96 & 0.00 & 0.00 & 0.00 & 0.00 & 1.22 \\
\hline $\mathrm{C} 22$ & 4.54 & 0.00 & 0.00 & 0.00 & 0.00 & 0.00 & 0.00 & 0.00 & 0.00 & 0.00 & 0.00 & 0.00 \\
\hline $\mathrm{C} 23$ & 0.00 & 0.00 & 0.00 & 0.00 & 0.00 & 0.00 & 0.00 & 0.00 & 0.00 & 0.00 & 0.00 & 0.00 \\
\hline $\mathrm{C} 24$ & 0.00 & 0.00 & 1.19 & 0.00 & 1.32 & 0.00 & 0.00 & 0.00 & 0.00 & 0.00 & 0.00 & 0.00 \\
\hline $\mathrm{C} 25$ & 1.16 & 0.00 & 0.00 & 0.00 & 0.00 & 0.00 & 0.00 & 0.00 & 0.00 & 0.00 & 0.00 & 0.00 \\
\hline $\mathrm{C} 26$ & 0.00 & 1.69 & 1.03 & 1.55 & 2.90 & 0.00 & 0.00 & 1.09 & 0.00 & 1.07 & 0.00 & 0.00 \\
\hline $\mathrm{C} 27$ & 1.96 & 0.00 & 0.00 & 0.00 & 0.00 & 0.00 & 0.00 & 0.00 & 0.00 & 0.00 & 0.00 & 0.00 \\
\hline $\mathrm{C} 28$ & 4.77 & 0.00 & 0.00 & 0.00 & 0.00 & 0.00 & 0.00 & 0.00 & 0.00 & 0.00 & 0.00 & 0.00 \\
\hline $\mathrm{C} 29$ & 1.39 & 0.00 & 0.00 & 0.00 & 0.00 & 0.00 & 0.00 & 0.00 & 0.00 & 0.00 & 0.00 & 0.00 \\
\hline C30 & 0.00 & 0.00 & 0.00 & 0.00 & 0.00 & 0.00 & 0.00 & 0.00 & 0.00 & 0.00 & 0.00 & 0.00 \\
\hline C31 & 0.00 & 0.00 & 0.00 & 0.00 & 0.00 & 0.00 & 0.00 & 0.00 & 0.00 & 0.00 & 0.00 & 0.00 \\
\hline $\mathrm{C} 32$ & 0.00 & 0.00 & 0.00 & 1.04 & 2.51 & 0.00 & 0.00 & 0.00 & 0.00 & 0.00 & 0.00 & 0.00 \\
\hline C33 & 0.00 & 0.00 & 0.00 & 1.10 & 0.00 & 0.00 & 0.00 & 0.00 & 0.00 & 0.00 & 0.00 & 0.00 \\
\hline C34 & 0.00 & 0.00 & 0.00 & 1.32 & 0.00 & 0.00 & 0.00 & 0.00 & 0.00 & 0.00 & 0.00 & 0.00 \\
\hline C35 & 0.00 & 0.00 & 0.00 & 1.55 & 0.00 & 0.00 & 0.00 & 0.00 & 0.00 & 0.00 & 0.00 & 0.00 \\
\hline C36 & 0.00 & 0.00 & 0.00 & 0.00 & 1.37 & 0.00 & 0.00 & 0.00 & 0.00 & 0.00 & 0.00 & 0.00 \\
\hline C37 & 4.18 & 0.00 & 0.00 & 0.00 & 1.12 & 0.00 & 0.00 & 0.00 & 0.00 & 0.00 & 0.00 & 0.00 \\
\hline C38 & 0.00 & 0.00 & 0.00 & 0.00 & 0.00 & 0.00 & 0.00 & 0.00 & 0.00 & 0.00 & 0.00 & 0.00 \\
\hline TOTAL & 82.58 & 27.36 & 16.96 & 15.21 & 17.32 & 8.15 & 11.54 & 8.56 & 8.16 & 7.43 & 7.85 & 5.86 \\
\hline Pri/Phy & 0.84 & 3.80 & 0.84 & 0.93 & 1.28 & 0.57 & 0.41 & 1.09 & 0.93 & 1.16 & 0.88 & 1.01 \\
\hline $\mathrm{CPI}_{25-35}$ & 0.78 & nd & nd & 0.35 & nd & nd & nd & nd & nd & nd & nd & nd \\
\hline $\mathrm{C}_{\max }$ & $\mathrm{C} 14$ & C14 & $\mathrm{C} 16$ & $\mathrm{C} 21$ & $\mathrm{C} 26$ & $\mathrm{C} 19$ & $\mathrm{C} 18$ & $\mathrm{C} 20$ & $\mathrm{C} 18$ & $\mathrm{C} 20$ & $\mathrm{C} 17$ & $\mathrm{C} 21$ \\
\hline C17/Pri & 0.26 & nd & 0.40 & 1.14 & 1.30 & nd & nd & nd & nd & nd & 1.82 & nd \\
\hline C18/Phy & 0.41 & nd & 0.36 & 0.72 & 0.61 & 0.59 & 0.87 & nd & 2.01 & nd & 0.72 & nd \\
\hline
\end{tabular}

The aliphatic hydrocarbons (AHCs) distribution in the samples was used to determine the hydrocarbon sources using isoprenoid (pristane/phytane) ratios, carbon preference index (CPI) and maximum carbon chain $\left(\mathrm{C}_{\max }\right)$.

The isoprenoid ratios are often used to differentiate the origin of hydrocarbons in environmental samples based on the fact that they are generally $<1$ for crude oil contaminated samples and $>1$ for uncontaminated samples (Wagener et al., 2010; Jeng and Huh, 2006; Pavlova and Papazova, 2003). The pristane/phytane ratios obtained from these samples indicated that petroleum products contributed to the hydrocarbon content of the soils within the plant vicinities. In HMA plant $\mathrm{A}$, the pristane/phytane ratios of subsurface samples 10 and $20 \mathrm{~m}$ in the dry season and $10 \mathrm{~m}$ in the rainy season were higher than unity. This suggests a mixture of oil and terrestrial plant materials which may be attributed to the decayed vegetation in the subsurface soils since the vicinity has been previously used as farmstead (Osuji and Achugasim, 2007). Isoprenoids (pristane and phytane) were not detected in samples from distances beyond $30 \mathrm{~m}$ in HMA Plant A during the dry season. Their presence in all samples from both HMA plants in the rainy season may be attributed to dissolutions and runoffs associated with the rains. The $n \mathrm{C}_{17}$ /pristane and $n \mathrm{C}_{18}$ /phytane ratios showed values below or close to 1 in most samples during the rainy season and above 1 in most dry season samples. Degradation process is suggested by values of $n \mathrm{C} 17 /$ pristane and $n \mathrm{C} 18 /$ phytane ratios below or close to 1 (Massone et al., 2013). This suggests that the rains enhance biodegradation of the hydrocarbons in the rainy season. The $n \mathrm{C}_{17} /$ pristane and 
$n \mathrm{C}_{18}$ /phytane ratios of the control samples in the rainy season may be attributed to the diverse sources of hydrocarbons in the area probably from leaching and runoffs associated with the rains.

Table 3: Aliphatic hydrocarbons concentration in HMA Plant B (Dry Season)

\begin{tabular}{|c|c|c|c|c|c|c|c|c|c|c|}
\hline \multirow{2}{*}{$\begin{array}{c}\text { AHCs } \\
(\mathrm{mg} / \mathrm{kg})\end{array}$} & \multicolumn{2}{|l|}{$10 \mathrm{~m}$} & \multicolumn{2}{|l|}{$20 m$} & \multicolumn{2}{|c|}{$30 \mathrm{~m}$} & $10 \mathrm{~m}$ & \multicolumn{3}{|c|}{$1 \mathrm{~km}$} \\
\hline & $\mathbf{S}$ & SS & $\mathbf{S}$ & SS & $\mathbf{S}$ & SS & $\mathbf{S}$ & SS & CS & CSS \\
\hline C9 & 0.00 & 0.00 & 1.12 & 0.00 & 0.00 & 0.00 & 0.00 & 0.00 & 0.00 & 0.00 \\
\hline $\mathrm{C} 10$ & 0.00 & 0.00 & 0.00 & 0.00 & 0.00 & 0.00 & 0.00 & 0.00 & 0.00 & 0.00 \\
\hline $\mathrm{C} 11$ & 0.00 & 0.00 & 0.00 & 0.00 & 0.00 & 0.00 & 0.00 & 0.00 & 0.00 & 0.00 \\
\hline $\mathrm{C} 12$ & 3.09 & 0.00 & 0.00 & 0.00 & 0.00 & 0.00 & 2.08 & 0.00 & 0.00 & 0.00 \\
\hline C13 & 6.47 & 0.00 & 0.00 & 0.00 & 0.00 & 3.13 & 2.46 & 0.00 & 0.00 & 0.00 \\
\hline $\mathrm{C} 14$ & 19.73 & 0.73 & 1.00 & 0.00 & 3.12 & 3.21 & 4.47 & 0.00 & 0.00 & 0.00 \\
\hline $\mathrm{C} 15$ & 27.29 & 1.81 & 2.60 & 0.00 & 2.13 & 1.82 & 7.42 & 0.00 & 0.00 & 0.00 \\
\hline $\mathrm{C} 16$ & 23.24 & 5.97 & 2.81 & 0.00 & 2.63 & 3.52 & 8.30 & 0.00 & 0.00 & 0.00 \\
\hline $\mathrm{C} 17$ & 9.71 & 14.84 & 3.43 & 0.00 & 5.19 & 1.75 & 7.73 & 2.56 & 0.00 & 0.00 \\
\hline Pristane & 14.97 & 5.03 & 0.43 & 0.00 & 5.62 & 3.13 & 6.15 & 0.38 & 0.00 & 0.00 \\
\hline C18 & 30.38 & 10.29 & 4.25 & 0.00 & 6.04 & 3.46 & 10.36 & 2.41 & 0.00 & 0.00 \\
\hline Phytane & 21.14 & 7.28 & 0.00 & 0.00 & 2.35 & 4.95 & 8.59 & 1.63 & 0.00 & 0.00 \\
\hline C19 & 53.27 & 11.53 & 5.63 & 0.00 & 14.54 & 3.63 & 13.31 & 0.78 & 0.00 & 0.00 \\
\hline $\mathrm{C} 20$ & 4.87 & 12.84 & 6.02 & 0.00 & 13.12 & 2.12 & 14.12 & 0.50 & 0.00 & 0.00 \\
\hline $\mathrm{C} 21$ & 10.98 & 6.76 & 5.94 & 0.42 & 3.13 & 1.46 & 12.49 & 0.40 & 0.43 & 0.00 \\
\hline $\mathrm{C} 22$ & 5.20 & 5.63 & 2.57 & 0.00 & 4.29 & 1.97 & 7.26 & 0.60 & 0.00 & 0.00 \\
\hline $\mathrm{C} 23$ & 12.44 & 1.38 & 2.12 & 0.00 & 4.18 & 3.01 & 7.42 & 0.64 & 0.00 & 0.00 \\
\hline $\mathrm{C} 24$ & 2.00 & 1.42 & 5.70 & 0.00 & 3.32 & 3.32 & 3.89 & 0.77 & 0.00 & 0.00 \\
\hline $\mathrm{C} 25$ & 8.64 & 1.16 & 5.92 & 0.00 & 2.60 & 5.10 & 18.26 & 0.84 & 0.00 & 0.00 \\
\hline $\mathrm{C} 26$ & 8.13 & 1.71 & 6.83 & 0.00 & 4.51 & 2.54 & 25.56 & 0.96 & 1.11 & 0.00 \\
\hline $\mathrm{C} 27$ & 4.04 & 1.75 & 9.49 & 1.13 & 4.59 & 1.15 & 13.71 & 1.10 & 1.25 & 1.16 \\
\hline $\mathrm{C} 28$ & 9.03 & 2.73 & 6.49 & 1.20 & 5.18 & 2.21 & 57.69 & 1.25 & 1.14 & 1.27 \\
\hline $\mathrm{C} 29$ & 41.28 & 3.85 & 4.42 & 1.37 & 5.92 & 1.67 & 15.81 & 2.00 & 1.70 & 1.61 \\
\hline C30 & 38.27 & 4.14 & 4.83 & 1.79 & 5.93 & 1.46 & 18.60 & 1.55 & 1.40 & 1.49 \\
\hline C31 & 4.61 & 1.40 & 2.22 & 0.00 & 1.38 & 3.37 & 17.47 & 1.42 & 1.36 & 0.00 \\
\hline C32 & 8.48 & 1.64 & 2.85 & 0.00 & 1.39 & 4.33 & 16.08 & 1.43 & 1.52 & 1.85 \\
\hline C33 & 5.77 & 2.13 & 1.99 & 0.00 & 0.00 & 3.35 & 20.53 & 2.11 & 1.38 & 1.76 \\
\hline C34 & 7.18 & 1.66 & 1.87 & 0.00 & 0.00 & 1.98 & 21.48 & 1.54 & 1.61 & 0.00 \\
\hline C35 & 1.89 & 1.77 & 1.38 & 0.00 & 0.00 & 1.31 & 5.09 & 1.22 & 1.68 & 0.00 \\
\hline C36 & 2.45 & 1.74 & 1.44 & 0.00 & 2.00 & 3.61 & 6.21 & 1.66 & 1.92 & 0.00 \\
\hline C37 & 2.18 & 2.12 & 2.73 & 0.00 & 3.26 & 3.93 & 7.27 & 2.04 & 3.33 & 0.00 \\
\hline C38 & 3.11 & 2.91 & 2.94 & 0.00 & 3.89 & 2.80 & 9.31 & 2.76 & 4.12 & 0.00 \\
\hline TOTAL & 389.84 & 116.22 & 99.02 & 5.91 & 110.31 & 79.29 & 369.12 & 32.55 & 23.95 & 9.14 \\
\hline Pri/Phy & 0.71 & 0.69 & nd & nd & 2.39 & 0.63 & 0.72 & 0.23 & nd & nd \\
\hline $\mathrm{CPI}_{25-35}$ & 0.93 & 0.83 & 0.87 & 0.84 & 0.71 & 1.06 & 0.65 & 0.99 & 0.97 & 0.98 \\
\hline $\mathrm{C}_{\max }$ & C19 & $\mathrm{C} 17$ & $\mathrm{C} 27$ & $\mathrm{C} 30$ & $\mathrm{C} 19$ & $\mathrm{C} 25$ & $\mathrm{C} 28$ & C38 & C38 & $\mathrm{C} 32$ \\
\hline C17/Pri & 0.65 & 2.95 & 7.98 & nd & 0.92 & 0.56 & 1.26 & 6.74 & nd & nd \\
\hline C18/Phy & 1.44 & 1.41 & nd & nd & 2.57 & 0.70 & 1.21 & 1.48 & nd & nd \\
\hline
\end{tabular}

The carbon preference index (CPI) of AHCs is useful for determining the degree of biogenic versus petrogenic input. It expresses the ratio of odd-carbon numbered to even-carbon numbered aliphatics in a given sample and has been used to differentiate biogenic AHCs from those of petrogenic or anthropogenic sources. CPI values $>2$ indicates predominant biogenic sources such as epicuticular waxes of terrestrial plants while CPI values of near unity signify the source as fossil fuels or incomplete combustion of petroleum products (Simoneit, 1984). The AHCs distribution of samples between $n \mathrm{C}_{24}-$ $n \mathrm{C}_{34}$ in both plants and both seasons showed that CPI values for both surface and subsurface soils were mostly below or close to unity signifying fossil fuel input as a result of vehicular emission and other anthropogenic activities (Kavouras et al., 1999). Subsurface sample $40 \mathrm{~m}$ from Plant A in the dry season has a CPI value of 2.57 which suggested multiple sources (petrogenic and biogenic) or a mixture of inputs (Bakhtiari et al., 2009; Azevedo et al., 2007). The CPI values of samples from both HMA plants indicate that inputs from anthropogenic activities were more significant than the input from biogenic sources (Didyk et al., 2000; Kauvoras et al., 1999). 
Table 4: Aliphatic hydrocarbons concentration in HMA Plant B (Rainy Season)

\begin{tabular}{|c|c|c|c|c|c|c|c|c|c|c|}
\hline \multirow{2}{*}{$\begin{array}{c}\text { AHCs } \\
(\mathbf{m g} / \mathbf{k g})\end{array}$} & \multirow{2}{*}{$\frac{10 \mathrm{~m}}{\mathrm{~S}}$} & \multicolumn{2}{|r|}{$20 \mathrm{~m}$} & \multicolumn{2}{|c|}{$30 \mathrm{~m}$} & \multicolumn{2}{|c|}{$40 \mathrm{~m}$} & \multicolumn{2}{|c|}{$1 \mathrm{KM}$} & \multirow[b]{2}{*}{ CSS } \\
\hline & & SS & $\mathbf{S}$ & SS & $\mathbf{S}$ & SS & $\mathbf{S}$ & SS & CS & \\
\hline C9 & 0.00 & 0.00 & 0.00 & 0.00 & 0.00 & 0.00 & 0.00 & 0.00 & 0.00 & 0.00 \\
\hline $\mathrm{C} 11$ & 0.00 & 0.00 & 0.00 & 0.00 & 0.00 & 0.00 & 0.00 & 0.00 & 0.00 & 0.00 \\
\hline $\mathrm{C} 12$ & 0.00 & 0.00 & 0.00 & 0.00 & 0.00 & 0.00 & 2.05 & 0.00 & 0.00 & 0.00 \\
\hline C15 & 1.60 & 0.00 & 0.00 & 0.00 & 0.00 & 0.00 & 0.00 & 0.00 & 0.00 & 0.00 \\
\hline $\mathrm{C} 16$ & 2.67 & 0.00 & 0.00 & 0.00 & 0.00 & 0.00 & 0.00 & 0.00 & 0.00 & 0.00 \\
\hline $\mathrm{C} 17$ & 2.35 & 0.00 & 0.00 & 0.00 & 0.00 & 0.00 & 1.94 & 0.00 & 4.64 & 0.00 \\
\hline Pristane & 4.61 & 3.76 & 3.01 & 1.20 & 3.78 & 7.69 & 10.40 & 3.48 & 1.06 & 2.48 \\
\hline $\mathrm{C} 21$ & 7.30 & 0.00 & 4.62 & 1.64 & 0.00 & 0.00 & 0.00 & 0.00 & 0.00 & 0.00 \\
\hline $\mathrm{C} 22$ & 2.04 & 0.00 & 7.09 & 0.00 & 0.00 & 0.00 & 0.00 & 0.00 & 0.00 & 0.00 \\
\hline $\mathrm{C} 23$ & 0.00 & 0.00 & 1.92 & 0.00 & 0.00 & 0.00 & 0.00 & 0.00 & 0.00 & 0.00 \\
\hline $\mathrm{C} 24$ & 0.00 & 1.68 & 0.00 & 0.00 & 1.35 & 1.87 & 0.00 & 2.31 & 0.00 & 1.83 \\
\hline $\mathrm{C} 25$ & 0.00 & 0.00 & 0.00 & 1.84 & 0.00 & 0.00 & 0.00 & 0.00 & 1.13 & 1.02 \\
\hline $\mathrm{C} 26$ & 0.00 & 7.26 & 0.00 & 0.00 & 3.17 & 0.00 & 1.32 & 0.00 & 0.00 & 0.00 \\
\hline $\mathrm{C} 27$ & 0.00 & 0.00 & 0.00 & 0.00 & 0.00 & 0.00 & 0.00 & 0.00 & 0.00 & 0.00 \\
\hline $\mathrm{C} 28$ & 0.00 & 0.00 & 0.00 & 0.00 & 0.00 & 0.00 & 2.52 & 0.00 & 0.00 & 0.00 \\
\hline $\mathrm{C} 29$ & 0.00 & 0.00 & 0.00 & 0.00 & 0.00 & 0.00 & 2.18 & 0.00 & 0.00 & 0.00 \\
\hline C36 & 0.00 & 0.00 & 0.00 & 0.00 & 0.00 & 0.00 & 0.00 & 0.00 & 0.00 & 0.00 \\
\hline C37 & 0.00 & 0.00 & 0.00 & 0.00 & 0.00 & 0.00 & 0.00 & 0.00 & 0.00 & 0.00 \\
\hline C38 & 0.00 & 0.00 & 0.00 & 0.00 & 0.00 & 0.00 & 0.00 & 0.00 & 0.00 & 0.00 \\
\hline TOTAL & 58.3 & 24.96 & 39.03 & 8.69 & 50.45 & 33.34 & 65.08 & 28.62 & 14.76 & 13.31 \\
\hline Pri/Phy & 0.40 & 0.45 & 0.40 & 0.62 & 0.41 & 0.82 & 1.59 & 0.59 & 0.72 & 0.53 \\
\hline$* \mathrm{CPI}_{25-35}$ & nd & nd & nd & nd & nd & nd & nd & nd & nd & nd \\
\hline $\mathrm{C}_{\max }$ & C19 & $\mathrm{C} 26$ & $\mathrm{C} 22$ & $\mathrm{C} 20$ & C34 & C19 & C19 & C19 & $\mathrm{C} 20$ & C18 \\
\hline C17/Pri & 0.51 & nd & nd & nd & nd & nd & 0.19 & nd & 4.38 & nd \\
\hline C18/Phy & 0.61 & nd & nd & nd & nd & nd & 0.64 & nd & 1.08 & 0.42 \\
\hline
\end{tabular}

*CPI; Carbon Preference Index $=0.5 \times\left[\left(\mathrm{C}_{25}+\mathrm{C}_{27}+\mathrm{C}_{29}+\mathrm{C}_{31}+\mathrm{C}_{33}\right)\right] /\left[\left(\mathrm{C}_{24}+\mathrm{C}_{26}+\mathrm{C}_{28}+\mathrm{C}_{30}+\mathrm{C}_{32}\right)\right]$ $\left.+\left[\left(\mathrm{C}_{25}+\mathrm{C}_{27}+\mathrm{C}_{29}+\mathrm{C}_{31}+\mathrm{C}_{33}\right)\right] /\left(\mathrm{C}_{24}+\mathrm{C}_{26}+\mathrm{C}_{28}+\mathrm{C}_{30}+\mathrm{C}_{32}+\mathrm{C}_{34}\right)\right], \mathbf{S}$; surface sample, $\mathbf{S S}$; subsurface sample, $\mathbf{C S}$; control surface sample, CSS; control subsurface sample, pri/phy; pristane/phytane, nd; cannot be detected because of the zero value of the numerator and/or denominator

The CPI of the rainy season samples could not be calculated because $n \mathrm{C}_{24}-n \mathrm{C}_{34}$ was not detected in the samples except for surface sample $10 \mathrm{~m}$ and subsurface sample $20 \mathrm{~m}$ in Plant A. Thus the AHCs in the rainy season samples might have been dominated by the short carbon chain aliphatics due to runoffs and predominance of hydrocarbons from diesel and petrol. The maximum AHCs concentration $\left(\mathrm{C}_{\max }\right)$ varied from $n \mathrm{C}_{16}-n \mathrm{C}_{35}$ in HMA Plant $\mathrm{A}$ in the dry season and from $n \mathrm{C}_{14}-n \mathrm{C}_{26}$ during the rainy season. In HMA Plant $\mathrm{B}$, it varied from $n \mathrm{C}_{19}-n \mathrm{C}_{38}$ during the dry season and $n \mathrm{C}_{19}-n \mathrm{C}_{34}$ during the rainy season. This was largely due to variations in the relative contribution of anthropogenic and biogenic sources. In the dry season, the samples closer to the asphalt plants peaked between $n \mathrm{C}_{16}-n \mathrm{C}_{19}$ indicating petrogenic input. The farther samples especially in Plant A were dominated by $n \mathrm{C}_{35}$ except for surface sample $50 \mathrm{~m}$ that peaked at $n \mathrm{C}_{27}$. While this might be attributed to easy susceptibility of short chain aliphatics to weathering, it also suggests biological input because high concentration of aliphatics dominated by odd number peaks is an indication of significant biological input (Maioli et al., 2011; Young and Wang, 2002).

The dry season samples in HMA Plant B also showed petrogenic sources more from lubricant use. 
Simoneit, (1986) has reported that a profile peak dominated by $n \mathrm{C}_{19}$ is an indication of lubricant oil used in gasoline and diesel powered engines. This was not surprising as the Plant B vicinity also serves as a maintenance yard for heavy duty vehicles and equipment. The rainy season samples of both plants showed clear domination of petrogenic inputs though there was reduced asphalt production in the rainy season. This might be ascribed to runoffs and dissolutions during rains as short chain carbons are more susceptible to weathering and redistribution during rainfall especially to areas that might have been predominantly dominated by biogenic sources.

Conclusion: The soil samples under study were polluted by aliphatic hydrocarbons when compared with the control samples. The most polluted sites were closer to the HMA plants which suggest anthropogenic activities associated with asphalt production as the major source of the hydrocarbons.

\section{REFERENCES}

Azevedo, DA; Goncalves, ML; Silva, DB (2007). Organic geochemistry of the Angra Dos Reis marine sediments: aliphatic and polycyclic aromatic hydrocarbons. Environ. Forensics, 8: 245-256

Bakhtiari, AR; Zakaria, MP; Yaziz, MI; Lajis, MNH; $\mathrm{Bi}, \mathrm{X}$ (2009). Polycyclic aromatic hydrocarbons and n-alkanes in suspended particulate matter and sediments for Langat River, Peninsular Malaysia. Environ. Asia. 2: 1-10

Didyk, BM; Simoneit, BRT; Pezoa, LA; Riveros, ML; Flores, AA (2000). Urban aerosol particles of Santiago, Chile: content and molecular characterisation. Atmos. Environ. 34: 1167-1179

EPA (2000). Hot mix asphalt plants emission assessment report. EPA-454/R-00-019. Office of Air Quality Planning and Standards. Research Triangle Park, NC 27111.

Ilechukwu, I (2013). Distribution and sources of hydrocarbons and bitumen derivatives in soils within the vicinity of two hot mix asphalt (HMA) plants. Ph.D Thesis. University of Port Harcourt, Rivers State, Nigeria

Jeng, WL; Huh, CA (2006). A comparison of sedimentary aliphatic hydrocarbon distribution between the Southern Okinawa Trough and a nearby River with high sediment discharge. Estuar. Coast Shelf Sci. 66: 217-224

Kassim, TA (2005). Forensic analysis and source partitioning of aliphatic hydrocarbon contamination in Lake Maruit aquatic sediments. Egypt. J. Aquat. Res. 31(2): 166-182
Kavouras, IG; Lawrence, J; Koutrakis, P; Stephanou, EG; Oyola, P (1999). Measurement of particulate aliphatic and polynuclear aromatic hydrocarbons in Santiago De Chile: source reconciliation and evaluation of sampling artifacts. Atmos. Environ. 33: 4977-4980

Maioli, OLG; Rodrigues, KC; Knoppers, BA; Azevedo, DA (2011). Distribution and sources of aliphatic and polycyclic aromatic hydrocarbons in suspended particulate matter from two estuarine systems. Cont. Shelf Res. 31: 11161127

Mamlouk, MS; Zaniewski, JP (2011). Materials for Civil and Construction Engineers. Pearson Education Inc. New Jersey.

Massone, CG; Wagener ALR; De Abreu HM; Veiga A (2013). Revisiting hydrocarbons source appraisal in sediments exposed to multiple inputs. Mar. Pollut. Bull. 73: 345-354

NIOSH (2001). Hazard Review; Health effects of occupational exposure to asphalt. National Institute for Occupational Safety and Health (DHHS) NIOSH Publication No. 2001-110

Osuji, LC; Achugasim, O (2007). Environmental degradation of polluting aromatic and aliphatic hydrocarbons: a case study. Chem. Biodivers. 4: 424-430

Pavlova, A; Papazova, G (2003). Oil spill identification by gas chromatography-mass spectrometry. J. Chromatogr. Sci. 41: 252-271

Rilwanu ML; Agbanure FE (2010). An assessment of the environmental impact of asphalt production in Nigeria. Anthropologist, 12(4): 277-287

Sakari, M; Zakaria, PM; Lajis, NH; Mohamed, CAR; Bahry, PS; Anita, SA; Chandru, K (2008). Characterisation, distribution, sources and origins of aliphatic hydrocarbons from surface sediment of Prai Strait, Penang, Malaysia: a widespread anthropogenic input. Environ. Asia. 2: $1-14$

Simoneit, BRT (1984). Application of molecular markers analysis to reconcile sources of carbonaceous particles in tropospheric aerosols. Sci. Total Environ. 36: 61-72

Simoneit, BRT (1986). Characterisation of organic constituents in aerosols in relation to their origin and transport: a review. Int. J. of Environ. Anal. Chem. 23: 207-237

Wagener, A; Hamacher, C; Farias, C; Marcus-Godoy, J; Scofield, A (2010). Evaluation of tools to 
identify hydrocarbon sources in recent and historical sediments of a Tropical Bay, Mar. Chem. 121: 67-69

Wang Z; Fingas M; Lambert P; Zeng G; Yang C; Hollebone B (2004). Characterisation and identification of the Detroit River mystery oil spill. J. Chromatogr. A. 1038: 201-214

Wang, Z; Fingas, M; Sergy, G (1995). Chemical characterisation of crude oil residues from Arctic Beach by GC/MS and GC/FID. Environ. Sci. Technol. 29: 2622-2631

Yang, C; Wang, Z; Yang, Z; Hollebone, B; Brown, C; Landriault, M; Fieldhouse, B (2011). Chemical Fingerprints of Alberta Oil Sands and Related Petroleum Products. Environ. Forensics. 12: $173-188$
Young, LH; Wang, SC (2002). Characterisation of nalkanes in $\mathrm{PM}_{2.5}$ of the Taipei aerosol. Atmos. Environ. 36: 477-482

Zeigler, C; MacNamarab, K; Wang, Z; Robbat, A. Jr (2008). Total alkylated polycyclic aromatic hydrocarbon characterization and quantitative comparison of selected ion monitoring versus full scan gas chromatography/mass spectrometry based on spectral deconvolution. J. Chromatogr. A. $1205: 109-116$

Zemo, AD; Bruya, EJ; Graf, ET (2007). The application of petroleum hydrocarbon fingerprint characterisation in site investigation and remediation. Ground Water Monit. Rem. 15(2): 146-156 